\title{
INTORNO ALL'ANALISI SINTATTICA DELLA FRASE SEMPLICE IN ITALIANO
}

Secondo una lunga tradizione confermata anche da opere illustri come la Sintassi italiana di Raffaello Fornaciari del 1881, gli autori delle grammatiche italiane, nel trattare la struttura della frase semplice, adottano una soluzione fortemente influenzata dalla semantica, con i cosiddetti complementi indiretti. Trattasi di un modello che si differenzia completamente da quello adottato nelle grammatiche delle altre grandi lingue europee, come il francese, il tedesco, il russo, in cui l'analisi della frase semplice è impostata su criteri essenzialmente sintattici.

Alcuni esempi possono subito chiarirci la differenza delle due impostazioni, quella semantica e quella sintattica. Prendiamo le seguenti frasi semplici:

1. La ringrazio del dono.

2. Egli tremava di paura.

3. Mi dispiace di non poter venire.

4. Il ragazzo ha fatto salti di gioia.

Secondo le grammatiche italiane abbiamo in queste frasi quattro complementi di causa, una determinazione che è formalmente esatta, ma non bastevole. Manca infatti un' analisi sintattica di questi complementi che deve precedere l'analisi semantica.

Un esame più approfondito ci fa vedere che questi quattro esempi racchiudono categorie sintattiche disparate e si rivelano come:

1. Oggetto indiretto

2. Determinazione circostanziale

3. Infinito in funzione di soggetto

4. Attributo

Alcuni altri esempi ci fanno vedere come l'importante determinazione sintattica debba precedere quella semantica:

1. Essi disputano di informatica - oggettoide di argomento

2. È un quadro di valore - attributo di qualità

3. Tremava di freddo - circostanziale di causa

4. È andato a caccia - oggettoide (o circostanziale) di fine

1. È bravo in linguistica - attributo di limitazione

2. Parlava in fretta - avverbiale di grado

3. Ha desistito dall'accusa - oggettoide di sep arazione

4. Fu trattenuto dalla polizia - oggettoide di agente 
Nota. - Com' è risaputo, il SINTAGMA è la connessione o combinazione di due o più segni correlati, di cui uno è l'elemento reggente e l'altro o gli altri sono elementi dipendenti. P. es.: un romanzo interessante, parlare ad alta voce. Il sintagma non ha funzione nella frase (ma può contenere in sè un elemento sintattico).

Diverso è il concetto di TASSEMA che rappresenta un elemento costitutivo della frase, portatore di funzioni logico-sintattiche. Tassema è un termine non ancora consolidato, usato tra altri dal Devoto, ma comunque utile.

Per gli studiosi di linguistica dei vari paesi europei l'analisi funzionale sintattica della frase assume un'importanza essenziale. Il grande grammatico tedesco Ulrich Engel, per esempio, persegue nella sua fondamentale grammatica (la Deutsche Grammatik) il tipo sintattico funzionale della frase e osserva che la sua opera potrebbe considerarsi una "grammatica della frase" (eine Satzgrammatik). Anche i grammatici di altre lingue europee danno un'importanza particolare all' aspetto sintattico della frase. Cosi, ad esempio, la Grammaire française di M. Breckx è tutta strutturata su questa analisi sintattica, per non parlare di altri grammatici che procedono in modo analogo.

Diverso è il modello di analisi della grammatica italiana. In essa, accanto alle strutture di base (soggetto e predicato) e altre poche strutture sintattiche (l'oggetto diretto, il complemento predicativo, l'attributo e l'apposizione) si riscontra un vasto raggruppamento di elementi chiamati con termine unico "complementi indiretti" e classificati secondo criteri semantici. Questi complementi indiretti, perlopiù introdotti da preposizioni, racchiudono in realta varie specie celate di funzioni sintattiche. Oggi i linguisti italiani avvertono sempre più l'insufficienza di fondamento di questi complementi indiretti ${ }^{1}$ il cui numero varia da 10 a 50 e più. L'impiego del termine di "funzione" in relazione a questi complementi è comumque un uso inadeguato. I complementi indiretti non hanno nulla a che fare con le funzioni sintattiche ${ }^{2}$.

\section{Proposte di innovazioni}

Le grammatiche italiane distinguono verbi transitivi e verbi intransitivi. Una INNOVAZIONE FONDAMENTALE della sintassi italiana, in accordo con la sintassi delle altre lingue europee, sarebbe lo sdoppiamento della funzione dei verbi transitivi con l'assegnazione ad essi di due compiti diversi.

1. Verbi transitivi diretti (che hanno per complemento un oggetto diretto, senza preposizione: vedo la casa, studio informatica).

2. Verbi transitivi indiretti (che introducono un oggetto indiretto, ossia preposizionale: discutono degli ultimi avvenimenti, si astengono dal farlo).

1 Serianni, Gramm., 99 (e altrove), Dardano-Trifone, Gramm., 113 (e altrove)

2 Si tratta di due livelli diversi 
Il confine tra verbi transitivi diretti, transitivi indiretti e intransitivi non è sempre preciso e talvolta uno stesso verbo può essere usato in modi diversi.

I. transitivo diretto:

provare un vestito

discutere una proposta

servire una vivanda

finire un lavoro

II. transitivo indiretto

provare a fare

discutere di astronomia

servire alla patria

finire di leggere

III. intransitivo

non proverò più

non voglio più discutere

servire da esempio

ho bell' e finito

L'oggetto indiretto

L'oggetto indiretto dei verbi transitivi indiretti si chiama anche OGGETTOIDE. Esso è un elemento costitutivo della frase, introdotto da preposizione.

Gli oggettoidi, di un numero imprecisato, vengono classificati secondo criteri semantici:

siamo entrati in casa - oggettoide di moto a luogo

si sono allontanati dal paese - moto da luogo

giravano per la piazza - moto per luogo

si attengono alle istruzioni - oggettoide dativale (di termine)

hanno discusso di tecnologia - oggettoide di argomento

lo hanno preso a servizio - oggettoide finale

li hanno forniti di tutto il necessario - oggettoide di mezzo

egli commercia in cereali - oggettoide di limitazione

si guardarono tra loro - oggettoide di reciprocanza

si è adirato con l'amico - oggettoide di relazione

Qui rientrano anche i costrutti infinitivi preposizionali:

spero di poter venire

credo di non arrivare in tempo

siamo certi di aver ragione

egli si ostina a negarlo

avete preparato da mangiare?

essi si apprestano a partire. 
Nota. - Anche certi aggettivi possono alle volte usarsi in funzione di oggettoide: gradevole all'udito, piacevole alla vista, abile alle armi.

I. verbi transitivi, sia diretti che indiretti, possono avere tre generi o forme verbali: 1. la diatesi attiva, 2. quella passiva e 3. la diatesi riflessiva.

Nelle grammatiche italiane odierne gli elementi funzionali che costituiscono la frase semplice, si riducono praticamente ai cinque già ricordati:

1. il soggetto

2. il predicato (nominale e verbale)

3. l'oggetto diretto

4. l'attributo

5. l'apposizione

Quanto al complemento predicativo, esso va considerato come una sottospecie del predicato nominale in cui il verbo essere è sostituito da verbi copulativi come sembrare, apparire (egli sembra contento, essi appaiono felici).

\section{I circostanziali}

Fra i componenti funzionali della frase semplice, non ricordati dalle grammatiche italiane, un'importanza rilevante spetta ai CIRCOSTANZIALI, una categoria di tassemi di cui tratta ampiamente, tra gli altri, Maurice Grevisse nel suo ben noto Le bon usage.

Trattasi di un costituente funzionale della frase che comprende varie specie semantiche rispondenti alle domande: dove?, quando?, in che occasione?, perche?, con chi?, con che cosa?, a che scopo?, con che conseguenze?

Ecco alcuni esempi di circostanziali:

Egli abita a Mantova - stato in luogo

Gabriele D'Annunzio è nato nel 1863 - tempo

Alla festa ho incontrato molti amici - occasione

Si è dimesso per motivi di età - causa

E partito con i suoi parenti - compagnia

Lo ha fatto a danno suo - conseguenza

Egli commercia in cereali - limitazione

Con questo tempo si viaggia bene - occasione

Senza biglietti non si entra - condizione supposta

Con grande disappunto l'ho appreso - conseguenza

Malgrado gli ostacoli ha ottenuto il posto - fatto non impediente la conseguenza

Alle volte il circostanziale può coincidere con l'oggettoide:

L'ho ringraziato del dono

In questo esempio il sintagma "del dono" può essere interpretato come oggetoide di causa o circostanziale di causa, ma simili casi sono rari. In linea generale vale la regola: quando la preposizione è legata al verbo, abbiamo un oggettoide, quando in- 
vece la preposizione fa parte integrante dell'espressione sintattica, abbiamo un circostanziale. Ecco alcuni esempi:
a) Oggettoidi
abbiamo discusso di sintassi
egli ha rinunciato al dono
si è adirato con lui
siamo passati per il ponte
b) Circostanziali
il ragazzo era pallido di paura
ci vedremo al ritorno
dorme con le finestre aperte verro per Natale

Per gran parte dei linguisti tra i circostanziali rientrano anche i cosiddetti AVVERBIALI che rispondono innanzi tutto alla domanda COME? P. es.: Egli progredisce bene. Si comportano conforme agli ordini. Sono questi avverbiali di modo che si possono far rientrare fra i circostanztali. Cosi come anche gli avverbiali di mezzo o strumento (abbiamo appreso la notizia per radio; il pane si prepara con la farina).

\section{L'a tributo}

Secondo una concezione tradizionale vigente nelle grammatiche italiane l' attributo è un aggettivo che qualifica e determina un nome da cui dipende sintatticamente. Esso segue il nome o lo precede.

In tempi più recenti questa concezione è stata un po' allentata e alcuni autori permettono che anche sintagmi preposizionali possano fungere da attributi. In tal modo anche un sintagma del tipo "uno studioso valoroso" può essere reso con un costrutto prepositivo: "e uno studioso di valore". Così Giacomo Devoto nelle sue "Lezioni prestrutturali" assegna un valore attributivo a strutture analitiche del tipo:

non era più un uomo "da averne paura" con un volto "tra l'attonito e l'adirato"

Nelle grammatiche delle altre grandi lingue europee l'attributo viene considerato come un tassema subordinato di forma semplice o complessa. Esso precisa o specifica un termine nominale della frase (un sostantivo, un aggettivo, un pronome, un avverbio o raramente una proposizione intera e qualche altro caso).

Possono compiere la funzione di attributo principalmente aggettivi, sostantivi e in misura molto minore pronomi, avverbi e proposizioni intere. Dalla varia combinazione di questi gruppi di parole risultano varie diecine di attributi che vengono esaminati qui di seguito.

3 Devoto, o.c., 125. 
I. Sostantivi più attributi

A) Sostantivo più aggettivo

E l'attributo tipico delle grammatiche italiane. Abitualmente è posposto, specie quando si vuole richiamare di più l'attenzione sull'aggettivo stesso. Possono compiere la funzione di attributo aggettivi qualificativi, indicativi, numerali, unitamente a participi. Tutte le grammatiche riportano regole sulla posizione dell'aggettivo attributivo e sulla natura dell'aggettivo limitativo.

Gli aggettivi sostantivati usati in qualita di epiteti rientrano in una categoria intermedia fra attributo e apposizione. P. es.: Alessandro il Grande, Lorenzo il Magnifico, Plinio il Giovane. Trattasi in fondo di attributi appositivi.

B) Sostantivi più sostantivi giustapposti

Si distinguono varie specie di questi attributi

a) Sostantivo che determina un altro sostantivo per giustapposizione: un treno merci, una nave ospedale, un cane lupo, il giornale radio, un decreto legge, la carta moneta, il capo gruppo, un tessuto fantasia

b) Rientrano in questa serie gli attributi di denominazione (vie, piazze, monti, fiumi): via Mazzini, piazza Garibaldi, monte Bianco, fiume Tevere.

Questo costrutto si usa anche con alcuni altri nomi comuni uniti a nomi propri: il poeta Omero, il padre Cristoforo, il pittore Raffaello, il dio Marte, il Palazzo Barberini, il generale Guerreschi.

Inoltre con i titoli: il dottor Carli, il professur Rossi, il re Umberto, pero: Federico Imperatore (in stile elevato).

Un caso particolare è rappresentato dal gruppo nome e cognome dove non tutti i linguisti si accordano a considerare il cognome come attributo appositivo: Ugo Foscolo, Guglielmo Marconi.

C) Sostantivi più sostantivi introdotti da preposizione

Questo tipo di attributo, assai numeroso, è semanticamente articolato in parecchi sottogruppi. Nelle grammatiche italiane attuali, trattandosi di un costrutto preposizionale, è tollerato come eccezione, $\mathrm{ma}$ in linea generale è relegato nella vasta categoria dei complementi indiretti.

Ecco comunque una serie di questi attributi sostantivali introdotti da preposizioni:

a) relazioni di luogo (stato, moto a, da, per): una casetta al mare, un viaggio a $\mathrm{Pa}$ lermo, un treno per Padova, il ritorno dal viaggio, il transito per la piazza, il diretto via Firenze;

b) relazioni di tempo (quando, quanto tempo): un viaggio ogni due anni, una corsa di un'ora, un appuntamento per domani;

c) causa (un grido di dolore, un colpo di fortuna, assente per motivi di salute);

d) fine, scopo (una casa di riposo, un libro di lettura, occhiali da sole); 
e) materia (una statua di marmo, un orologio d'oro);

f) contenuto, argomento (una bottiglia di vino, un manuale di chimica)

g) specie (il manuale di fisica nucleare, un biglietto di banca, l'ufficio di giudice)

h) possesso (la casa del contadino, il cane del cacciatore, il balcone del palazzo, la porta dello scompartimento)

i) totalita divisa (una meta della somma, una parte della popolazione, un chilo di mele)

1) misura, estensione, età (un appartamento di cinque stanze, uno spazio di otto metri, una strada di tre chilometri, un ragazzo di dieci anni)

m) base, modello (un abito su misura, un premio secondo il merito)

Attributo soggettivo: l'aratura del contadino, il tramonto del sole, lo scioglimento delle nevi, il sonno della natura, il ristagno degli affari.

Attributo oggettivo: l'aratura dei campi, lo studio delle lingue, il consumo di energia, la coltura della mente, la paura della morte.

Attributi predicativi: l'agenzia d'informazione, la casa di spedizione, una società di assicurazione.

D) Sostantivi più altri elementi

a) sostantivo più pronome: $i$ nostri amici, i miei conoscenti, le osservazioni di lui, $i$ ricordi di loro, una sorpresa per voi, le sue possibilità;

b) sostantivi più infinito: la volontà di vincere, la difficoltà di procedere, non abbiamo tempo da perdere, il desiderio di conoscere, azioni da non fare;

c) sostantivo più avverbi: la gente di qui, i giovani di oggi, il compito per domani, $i$ denti davanti, quella via in fondo;

d) sostantivi più nessi sintattici: una notizia da non dimenticare, stoffe a vividi colori, un riso a denti stretti, un combattimento a corpo a corpo;

e) sostantivo più proposizione: una tipica situazione del si salvi chi può, un'informazione sul come stanno le cose;

Al gruppo e) appartengono anche le proposizioni relative attributive: gli spettatori che lo desiderano possono anche restare in sala, conosco il paese donde Lei proviene, il giovane che studia e degno di lode.

\section{Aggettivi più attributi}

1) Aggettivi più aggettivi: stanco morto, grigio verde, rosso scuro, lungo disteso, ubriaco fradicio, verde chiaro.

2) Aggettivi più sostsntivi (sostantivi che determinano aggettivi):

a) giustapposti: biondo oro, verde bottiglia, grigio perla, nero fumo, lontano tre chilometri, largo due metri;

b) introdotti da preposizioni o avverbi: degno di stima, contento del dono, pallido dallo spavento;

3) Aggettivo più pronomi: simile a lui, sicuro di ciò, più intelligente di te, molti di voi; 
4) Aggettivo più infinito: facile a capire, contento di ascoltare, pronto a partire, incapace di muoversi;

5) Aggettivo più avverbio (attributi avverbiali): molto bravo, estremamente interessante, meno prezioso, alquanto stanco;

6) Aggettivo più nesso sintattico: forte come un leone, rapido a guisa di vento;

7) Aggettivo e frase intera: contento come se avesse vinto un premio.

III. Pronome più attributo

Qualcuno di noi, niente di straordinario, alcuni di loro, noi docenti e voi studenti, nessuno dei due.

IV. Avverbi determinati da un attributo

Abbastanza bene, molto male, quasi niente, poco prima, prima di altro, a destra del palazzo.

V. Frasi intere determinate da atributo

Un energico "fate attenzione", un disperato "si salvi chi può".

Nota 1. - Abbiamo visto che il complemento predicativo (chiamato anche predicativo legato) rappresenta solo una sottospecie del predicato nominale e viene introdotto da verbi copulativi (sembrare, parere, fungere, ecc.). Accanto a questo tipo di predicativo, in tempi recenti è stato costituito il cosiddetto PREDICATIVO LIBERO che viene introdotto da verbi predicativi. Esso rappresenta un vero tassema, cioè un elemento costitutivo della frase e richiede un trattamento particolare. C'è poi un altro tassema, il GIUDICATIVO, un nesso del tipo tutto sommato, secondo la mia opinione, che è ancora in fase di evoluzione. Ambedue questi costitutivi della frase vanno trattati a parte.

Nota 2. - Il testo di T. Alisova (v. la Bibliografia) non ostante il titolo, si differenzia completamente dall'impostazione sintattica del nostro contributo.

Nota 3. - Molti esempi, specie nella seconda parte del nostro articolo, sono tolti dalla Grammatica italiana descrittiva di cui sono coautore con Moritz Regula, una grammatica che in linea generale persegue le strutture sintattiche. 


\section{Bibliografia}

Alisova, T., Strutture semantiche e sintattiche della frase semplice in italiano, Firenze, 1972.

Altieri-Biagi, M. L. - Herlmann, L., Dalla lingua alla grammatica, Milano, 1974

Battaglia, S. - Pernicone, P., La grammatica italiana, Torino, 1957.

BINI, A., Nuova grammatica italiana, Firenze, 1941.

Bonfante, G. - Ferrero, P., Grammatica italiana per la scuola media, Milano,1972.

BRECKX, M., Grammaire française, Bruxelles, 1996.

ColI, P. L. - Rossi,G., Dolce idioma, Firenze, 1941.

Dardano, M. - Trifone, P., Grammatica italiana, Milano, 2001.

DEVOTO, G., Lezioni di sintassi prestrutturale, Firenze, 1974.

Duro, A., Grammatica italiana, Torino, 1957.

ENGEL, U., Deutsche Grammatik, Heidelberg, 1991.

ForNaCIARI, R., Sintassi italiana dell'uso moderno, Firenze, 1881.

GREVISSE, M., Le bon usage, Paris-Grenoble, 1986.

LE GoFFIC, P., Grammaire de la phrase française, Paris, 1993.

MigliorinI, B., Grammatica italiana, Firenze, 1958.

Nencioni, G., Saggi e memorie, Firenze, 2000.

REGULA, M. - JeRNEJ, J., Grammatica italiana descrittiva, Bern, 1974.

RENZI, L., - SALVI, G., Grande grammatica italiana di consultazione, I-III, Bologna, 1989-1999.

SERIANNI, L., Grammatica italiana, Torino, 1991.

TrabalzA, G. - Allodoli, E., La grammatica degli Italiani, Firenze, 1939. 


\section{Povzetek}

\section{K SKLADENJSKI ANALIZI PROSTEGA STAVKA V ITALIJANŠČINI}

$V$ italijanskih slovnicah je skladenjska analiza stavka največkrat zasnovana na semantiki, pri tem pa se zanemarja vloga predlogov. Ta pristop je precej drugačen $v$ slovnicah najbolj obravnavanih evropskih jezikov, kjer je poudarek ravno na analizi skladenjske ravní in ima pomenskost mnogo manj teže.

Splošno sprejeti italijanski model predstavlja predložne sintagme v obsežnem pomenskem sklo$\mathrm{pu}$; imenuje jih complementi indiretti 'nepremi predmeti'. 